\title{
NEW NICE GUIDELINES AIM TO IMPROVE QUALITY OF DENTISTRY PROVISION IN THE UK
}

The British Dental Health Foundation (BDHF) has welcomed the focus on giving patients the ability to make an informed decision about their care as outlined in the new NICE guidelines on oral health promotion in general dental practice.

The guidelines cover how general dental practice teams can communicate advice about oral hygiene, the use of fluoride, as well as how areas such as diet, smoking, smokeless tobacco and alcohol intake affect oral health in order to help patients make informed decisions on their own care and encourage preventive treatments.

The NICE guidelines include lots of helpful information for dental professionals to help them to communicate with patients, such as how they can use appropriate words to discuss the strength of their recommendations.
The guidelines also include information about prescribing medicines, professional guidelines, standards and laws (including on consent and mental capacity), and safeguarding to help improve patient support.

Dr Ben Atkins, a dentist and Trustee of the British Dental Health Foundation, was a member of the committee for these NICE guidelines.

Dr Atkins said: 'These guidelines have been developed with the patient firmly in mind; they outline a patient-centred approach to ensure patients who are using the services are actively involved in discussions and able to make informed decisions about their care.

'Throughout the consultation stage it was recognised that interventions need to provide patients with support to help them change their behaviour in order to effectively change how they look after their oral health at all times.

'By focusing on providing staff in dental practices with the means to do this by following these guidelines we are hoping to see the quality of dental provision in the UK improve in the future.'

\section{BOOK REVIEW}

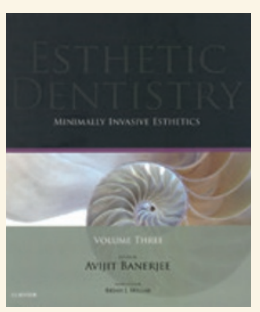

\section{ESSENTIALS OF ESTHETIC DENTISTRY: MINIMALLY INVASIVE ESTHETICS}

Volume Three

Avijit Banerjee (ed)

Elsevier

price $€ 61.99$ pp 344

ISBN 9780723455561

This book aims to introduce readers to the concept of minimally invasive dentistry, providing clinical tips and techniques that allow the reader to expand their clinical practice. Applicable to undergraduates, general practitioners and specialists alike, this easy-to-read text enriches the reader's knowledge of the aetiology and minimally invasive approach to managing a range of common conditions that may require aesthetic intervention, such as tooth discolouration, hypoplastic conditions and tooth wear.

With contributions from authors who are internationally renowned for their expertise in the field of minimally invasive aesthetic dentistry, the book is extensively referenced giving clinicians evidence based, ethical management strategies to restore and protect their patient's dentition. The overwhelming ethos of the book is that of tissue and pulpal preservation with a focus on early diagnosis and intervention.

The book is divided into well-structured sections taking the reader on a journey beginning with the diagnosis and treatment options of common clinical conditions that may require aesthetic intervention. It goes on to describe three of the most common minimally invasive management strategies: dental bleaching, adhesive composite restorations and direct/indirect tooth replacement options.

The authors approach subject matter consistently by giving background information, detailing indications and contraindications of each management strategy. Dental materials are discussed and step-by-step protocols guide the reader through the techniques. Packed full of clinical tips and sequential treatment photographs, this book allows any clinician to gain the knowledge and confidence to carry out these techniques.

Each chapter is accompanied by real clinical cases that are illustrated by colour clinical photographs and radiographs. They conclude with suggestions for further reading and often share questions that a patient may frequently ask about the treatment, accompanied by suggested answers. This highlights the importance of patient communication and managing expectations when embarking on aesthetic treatment.

The relaxed, engaging tone, logical structure, rich illustrations and quick reference boxes make this text an invaluable reference source which challenges our attitudes and current approach to managing aesthetic problems, whilst supporting us to make changes to our clinical practice. Both a practical guide and a summary of current literature, I feel this book is a triumph, championing the current shift in the teaching of minimally invasive dentistry and is a must have for any clinician who provides restorative treatment for both children and adults.

\section{FROM THE ARCHIVE}

Money is always a relatively sensitive topic. The GDC's decision to keep the ARF astronomically high for dentists has been well documented in these pages. Here's an item from the $B D J$ on 21 September, 1965 that sets out what the councils of the Medical Defence Union, Medical Protection Society and Medical and Dental Defence Union describes as 'steeply rising costs'. The narrative remains the same as today - more litigation, more legal fees and a need to maintain financial resources at a level "commensurate with future liabilities'.

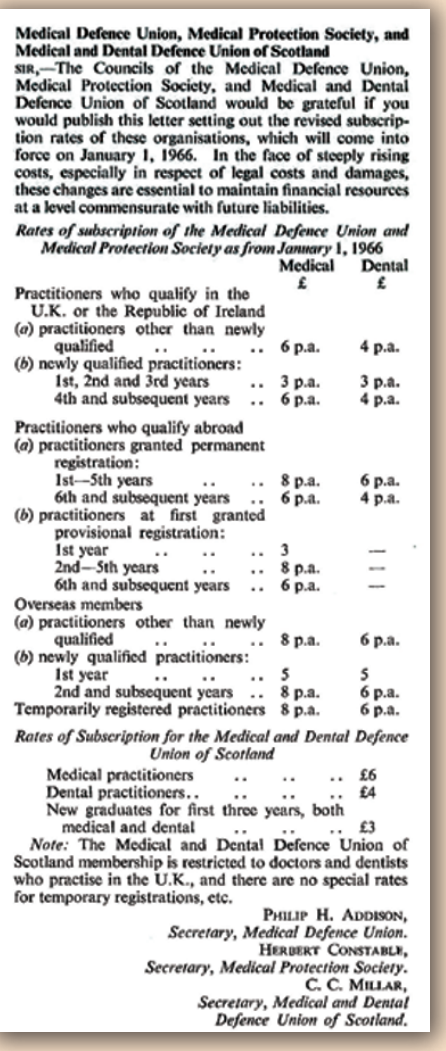

